\title{
CORRELAÇÃO ENTRE SOBREPESO, OBESIDADE E HIPERGLICEMIA EM MANAUS-AM
}

\author{
Maykom de Lira Barbosa ${ }^{1}$, Danielle Novais Antunes ${ }^{2}$, Leandro Negrelli ${ }^{3}$, Yara Ayami \\ Mattos Abe ${ }^{4}$, Rosany Piccolotto Carvalho ${ }^{5}$ \\ ${ }^{1}$ Graduando em Medicina pela Universidade Federal do Amazonas (UFAM), Faculdade de \\ Medicina, Manaus, Amazonas - Brasil. E-mail: maykomlira@gmail.com. \\ ${ }^{2}$ Graduanda em Medicina pela Universidade Federal do Amazonas (UFAM), Faculdade de \\ Medicina, Manaus, Amazonas - Brasil. \\ ${ }^{3}$ Graduando em Medicina pela Universidade Federal do Amazonas (UFAM), Faculdade de \\ Medicina, Manaus, Amazonas - Brasil. \\ ${ }^{4}$ Graduanda em Fisioterapia pela Universidade Federal do Amazonas (UFAM), Faculdade \\ de Fisioterapia e Educação Física, Manaus, Amazonas - Brasil. \\ ${ }^{5}$ Professora Doutora da Universidade Federal do Amazonas (UFAM), Departamento de \\ Ciências Fisiológicas, Manaus, Amazonas - Brasil.
}

Recebido em: 04/10/2019 - Aprovado em: 30/11/2019 - Publicado em: 15/12/2019 DOI: 10.18677/EnciBio_2019B15

\begin{abstract}
RESUMO
A obesidade é considerada um dos maiores problemas de saúde pública do mundo, sendo uma doença complexa e multifatorial, tal doença é muito associada ao surgimento de diversas doenças crônicas, entre elas o diabetes mellitus do tipo dois. O objetivo do presente estudo foi comparar grupos de indivíduos de diferentes idades e gêneros em Manaus por avaliação antropométrica e glicemia capilar. Tratase de um estudo descritivo transversal. Quanto à metodologia, utilizou-se glicosímetro portátil, balança e estadiômetro. Os participantes foram divididos em 10 grupos de 30 indivíduos, sendo 5 grupos masculinos divididos por faixa etária (G2 18-24 anos, G4 25-44 anos, G6 45-54 anos, G8 55-64 anos e G10 $\geq 65$ anos), e 5 grupos femininos divididos mesma forma e a glicemia foi avaliada pelo glicosímetro portátil. Quanto aos resultados, a média e desvio padrão do Índice de Massa Corpórea para os grupos foram G1 $(22,08 \pm 3,10)$, G2 $(25,2 \pm 3,73)$; G3 $(26,68 \pm 5,00)$; G4 $(28,37 \pm 3,77) ; \quad$ G5 $(28,53 \pm 4,46) ; \quad$ G6 $(28,45 \pm 3,06) ; \quad$ G7 $(29,3 \pm 4,20) ; \quad$ G8 $(28,58 \pm 4,32)$; $G 9(28,34 \pm 4,86)$ e G10 $(28,06 \pm 3,32)$. O sobrepeso foi observado em $64,6 \%$ das mulheres e $78,1 \%$ para homens. Aproximadamente $6 \%$ do total da amostra tinha hiperglicemia, dos quais todos tinham idade maior ou igual a 45 anos de ambos os sexos. Portanto, a amostra obtida reflete as altas taxas de sobrepeso e obesidade da cidade de Manaus, sendo mais prevalente entre os homens mais idosos, bem como uma maior taxa de hiperglicemia em indivíduos acima de 50 anos.

PALAVRAS-CHAVE: Diabetes Mellitus Tipo 2, Índice Glicêmico, Obesidade.
\end{abstract}

\section{CORRELATION AMONG OVERWEIGHT, OBESITY AND HYPERGLYCEMIA IN MANAUS-AM}

\section{ABSTRACT}

Obesity is considered two of the main public health problems in the world, being a complex and multifactorial disease, which is closely associated with the onset of 
several chronic diseases, including diabetes mellitus type two. The aim of this study is to compare groups of individuals of different ages and genders in Manaus by anthropometric assessment and capillary glucose. This is a cross-sectional descriptive study. As for methodology, were used portable glucometer, scale and stadiometer. Participants were divided into 10 groups of 30 individuals, 5 male groups divided by age group (G2 18-24 years, G4 25-44 years, G6 45-54 years, G8 55-64 years and $\mathrm{G} 10 \geq 65$ years) and others 5 female groups were similarly divide and the blood glucose were evaluated by portable glucometer. The results, mean and standard deviation of Body Mass Index for the groups were G1 (22.08 \pm 3.10$)$, G2

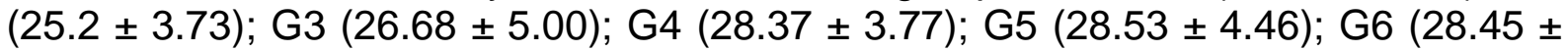
3.06); G7 (29.3 \pm 4.20$)$; G8 (28.58 \pm 4.32$) ; \mathrm{G9}(28.34 \pm 4.86)$ and G10 (28.06 \pm 3.32$)$. The overweight was observed in $64.6 \%$ of women and $78.1 \%$ in men. Approximately $6 \%$ of the total showed hyperglycemia, them aged 45 years and over of both genders. Therefore, it demonstrably reflects the high rates of overweight and obesity in the city of Manaus, being more prevalent among the older males, as well as one of the main rate of hyperglycemia in individuals over 50 years old.

KEYWORDS: Glycemic Index, Obesity, Type 2 Diabetes Mellitus.

\section{INTRODUÇÃO}

A obesidade é uma doença complexa, multifatorial e evitável (HRUBY; HU, 2015), podendo ser causada por distúrbios alimentares, relacionados a características culturais, socioeconômicas e familiares ou, menos frequentemente, por distúrbios endócrinos e doenças genéticas, como as síndromes de Prader-Willi e Laurence-Moon-Biedl (SCHWARTZ et al., 2017).

No Brasil, a obesidade vem crescendo demasiadamente, levantamentos apontam que mais de $50 \%$ da população está acima do peso, ou seja, na faixa de sobrepeso e obesidade. Entre crianças, estaria em torno de $15 \%$, segundo 0 Instituto Brasileiro de Geografia e Estatística - IBGE para o ano de 2014 (ISER et al., 2016).

Os indivíduos obesos têm risco aumentado de morbidade de doenças, tais como: depressão, diabetes mellitus tipo 2 (DM 2), doença cardiovascular e alguns tipos de câncer, como de endométrio, mama, próstata e cólon (ABRANCHES et al., 2015). Obesos apresentam pelo menos o dobro de chance de desenvolver resistência à insulina, dislipidemia, hipertensão, doenças coronarianas e reumatológicas. Essas associações e o crescente aumento da prevalência de obesidade, além de outros fatores de risco para doenças crônicas não transmissíveis, como inatividade física, tabagismo, pressão e colesterol elevados são responsáveis por uma grande quantidade de gastos em saúde (ORGUBY; HU, 2015).

Com o aumento da prevalência da obesidade, o número de pacientes com DM 2 tem aumentado concomitantemente ao número de obesos em todo o mundo. O desenvolvimento do DM 2 é, geralmente, o resultado de defeitos na produção ou na ação da insulina. Os pacientes portadores de diabetes desenvolvem descompensação das células pancreáticas, consequentemente, hiperglicemia (ABRANCHES et al., 2015).

No Brasil é realizado anualmente um inquérito telefônico pelo Sistema de Vigilância de Fatores de Risco e Proteção para Doenças Crônicas por Inquérito Telefônico - VIGITEL, pelo qual os indivíduos acima de 18 anos são questionados 
sobre índice de massa corpórea (peso e altura), idade, nível educacional, aspectos socioeconômicos e hábitos alimentares (BRASIL, 2018). Porém, é um estudo que apresenta viés ao contatar apenas indivíduos que possuem linha telefônica ativa e depende da declaração de quem participa, sem dispor de meios para confirmar os dados obtidos por meio da entrevista quanto ao índice de massa corpórea (IMC) e glicemia (ISER et al., 2016).

Nesse sentido, objetivou-se com o presente estudo avaliar em um grupo de 300 indivíduos a prevalência de obesidade, sobrepeso e diabetes no município de Manaus, realizando uma comparação entre grupos de indivíduos de diferentes faixas etárias e gêneros, ao avaliar IMC, índice glicêmico e determinar um panorama geral dos hábitos de vida e alimentares dos habitantes.

\section{MATERIAL E MÉTODOS}

\section{Amostragem}

Tratou-se de um estudo observacional transversal, cuja base populacional foi constituída por habitantes do município de Manaus de diferentes gêneros e faixas etárias. Foi realizado no município de Manaus, capital do estado do Amazonas. Foram incluídos 300 indivíduos, sendo um grupo de idosos de um projeto de extensão vinculado à Universidade Federal do Amazonas, além de funcionários de uma empresa e uma avaliação aleatória da comunidade em um local público. Foram abordados 300 indivíduos de diferentes gêneros (masculino e feminino), sendo a faixa etária pretendida acima de 18 anos, sendo divididos por faixa etária e gênero nos grupos demonstrados no quadro 1. Foram excluídos pacientes em tratamento com hipoglicemiantes, gestantes e pacientes ascíticos.

Todos os indivíduos foram submetidos ao Termo de Consentimento Livre e Esclarecidos - TCLE. Os procedimentos foram realizados conforme à resolução 466/12 do Conselho Nacional de Saúde, respeitando os preceitos éticos aos indivíduos participantes das pesquisas científicas. O projeto foi aprovado pelo Comitê de Ética e Pesquisa da Universidade Federal do Amazonas (CEP/UFAM), com o Certificado de Apresentação para Apreciação Ética - CAAE 92510918.0.0000.5020, parecer número 2.974.471.

QUADRO 1 Grupos relacionados por faixa etária e gêneros.

\begin{tabular}{|c|c|c|}
\hline GRUPO & GÊNERO & FAIXA ETÁRIA \\
\hline G1 우 & FEMININO & Entre 18 a 24 anos \\
\hline G20 & MASCULINO & Entre 18 a 24 anos \\
\hline G3우 & FEMININO & Entre 25 a 44 anos \\
\hline G40 & MASCULINO & Entre 25 a 44 anos \\
\hline G5우 & FEMININO & Entre 45 a 54 anos \\
\hline G6o & MASCULINO & Entre 45 a 54 anos \\
\hline G7우 & FEMININO & Entre 55 a 64 anos \\
\hline G80 & MASCULINO & Entre 55 a 64 anos \\
\hline G9우 & FEMININO & Maior ou igual a 65 anos \\
\hline $\mathrm{G} 100^{x}$ & MASCULINO & Maior ou igual a 65 anos \\
\hline
\end{tabular}

Fonte: Autores (2019) 


\section{Avaliação antropométrica}

A avaliação antropométrica foi realizada por meio das medidas de peso e estatura. Para avaliação do peso foi utilizada uma balança mecânica e para estatura uma estadiômetro. Os participantes estavam com roupas leves e descalços. A avaliação do estado nutricional foi realizada por meio da análise do IMC, que utiliza o peso em quilos dos indivíduos e o quadrado de sua altura em metros, sendo expresso em $\mathrm{kg} / \mathrm{m}^{2}$. Os pontos de corte para atribuição da classificação do estado nutricional segundo esse indicador são os estipulados pela Organização Mundial de Saúde (WHO, 1995), que preconiza como normais ou eutrófico os indivíduos que possuam índice de massa corpórea entre 18,5 e $24,9 \mathrm{k} / \mathrm{m}^{2}$, com baixo peso os indivíduos com índice de massa corpórea igual ou inferior a $18,4 \mathrm{k} / \mathrm{m}^{2}$, com sobrepeso aqueles com índice de massa corpórea entre 25 e $29,9 \mathrm{Kg} / \mathrm{m}^{2}$ e com obesidade aqueles com índice de massa corpórea superior a $30 \mathrm{~kg} / \mathrm{m}^{2}$ (quadro 2).

QUADRO 2 Classificação por Índice de massa corpórea (IMC).

\section{CLASSIFICAÇÃO}

Baixo peso

Peso normal

Sobrepeso

Pré-obeso

Obeso I

Obeso II

Obeso III
IMC (KG/M $)$

Baixo

$18,5-24,9$

$\geq 25$

25,0 a 29,9

30,0 a 34,9

$35,0-39,9$

$\geq 40,0$

RISCO DE COMORBIDADES

Fonte: Adaptada de World Health Organization (WHO), 1995.

\section{Avaliação glicêmica}

Para análise da glicemia dos participantes foi utilizado um glicosímetro portátil, com base nos índices da sociedade brasileira de diabetes, considerando diabetes mellitus com glicemia capilar casual com $200 \mathrm{mg} / \mathrm{dl}$ ou maior com sintomas clássicos. Para avaliação criteriosa e segura da glicemia capilar, foram utilizados os seguintes materiais: 1 par de luvas de procedimento para o pesquisador, 1 Aparelho dosador de glicemia (glicosímetro Accu-Check Active), 2 bolas de algodão embebidas em solução alcoólica a 70\%, 1 Tira-teste para o aparelho de glicemia, 1 Bandeja, 1 Lanceta para cada indivíduo com trava de segurança retrátil Safe T-Pro-Uno e 1 caixa para descarte de materiais perfurocortantes e/ou contaminados. 


\section{Análises estatísticas}

Os resultados foram expressos através da Média \pm desvio padrão (DP) para cada grupo de indivíduos o número $(n)$ foi indicado nas tabelas. As análises estatísticas utilizaram a Análise única de variância (ANOVA) e teste post-hoc de Tukey. O teste de $t$ student foi utilizado para a determinação da diferença significativa. O nível de significância adotado para os testes foi de $5 \%(p<0,05)$.

\section{RESULTADOS E DISCUSSÃO}

No período de outubro de 2018 a junho de 2019 foram avaliados 300 indivíduos que se encaixavam nos critérios de inclusão e aceitaram participar da pesquisa mediante assinatura do Termo de Consentimento Livre e Esclarecido (TCLE). Foram avaliadas massa corpórea, altura e glicemia capilar. Aproximadamente $71,7 \%$ dos voluntários apresentavam IMC maior ou igual a 25 (tabela 1).

TABELA 1 Número de indivíduos por faixa de IMC

\begin{tabular}{ccccc}
\hline GRUPOS & $\mathbf{1 8 - 2 4 , 9}$ & $\mathbf{2 5 - 2 9 , 9}$ & $\mathbf{3 0 - 3 9 , 9}$ & $\geq \mathbf{4 0}$ \\
\hline G1 & 24 & 6 & 0 & 0 \\
G2 & 15 & 13 & 2 & 0 \\
G3 & 10 & 12 & 7 & 1 \\
G4 & 5 & 13 & 12 & 0 \\
G5 & 6 & 14 & 10 & 0 \\
G6 & 3 & 20 & 7 & 0 \\
G7 & 5 & 13 & 12 & 0 \\
G8 & 6 & 12 & 12 & 0 \\
G9 & 7 & 12 & 10 & 1 \\
G10 & 4 & 18 & 8 & 0 \\
TOTAL & $85(28,33 \%)$ & $133(44,33 \%)$ & $80(26,67 \%)$ & $2(0,66 \%)$ \\
\hline
\end{tabular}

Os grupos de faixa etária entre 18 e 44 do sexo feminino tiveram uma média maior de IMC normal $(p<0,05)$. O grupo G1 apresentou a menor média de IMC (tabela 2), evidenciando que as mulheres mais jovens avaliadas foram as que tiveram menores taxas de IMC, em contrapartida, os indivíduos do sexo masculino da mesma faixa etária do grupo G2 tiveram média de IMC de 25,2. Já os indivíduos acima de 24 anos de ambos os sexos tiveram média entre 26 e 30 de IMC. Houve uma prevalência de aproximadamente $27,3 \%$ de obesidade, um número próximo de $23,0 \%$, o qual foi indicado pelos dados fornecidos pela VIGITEL do ano de 2018 para o município de Manaus, com maior número de indivíduos com obesidade nos grupos G4 (Masculino, 25-44 anos) e G7 (Feminino, 55-64 anos), ambos com 12 indivíduos (BRASIL, 2018).

$\mathrm{Na}$ região norte do país, segundo dados da Associação Brasileira para o Estudo da Obesidade e Síndrome Metabólica (ABESO, 2016), em torno de 25\% da população com cinco a nove anos de idade estão com excesso de peso, $17 \%$ entre 10 e 19 anos está com sobrepeso e 47,2\% dos adultos estão também com excesso de peso.

Pela análise estatística na tabela 2, ao comparar os grupos foi possível observar que os indivíduos do sexo masculino tiveram maiores IMC's $(p<0,05)$. Há 
estudos que evidenciam a maior prevalência em homens observada em diversos países, tais como no Estados Unidos, Japão e Nigéria (HSIA; HEYMSFIELD, 2018).

\begin{tabular}{ccccc}
\hline GRUPOS & MÉDIA & DP & MÍNIMO & MÁXIMO \\
\hline G1 & 22,08 & $\pm 3,10^{\mathrm{a}}$ & 18,04 & 29,97 \\
G2 & 25,20 & $\pm 3,73^{\mathrm{a}}$ & 19,17 & 33,10 \\
G3 & 26,68 & $\pm 5,00^{\mathrm{b}}$ & 18,10 & 41,67 \\
G4 & 28,38 & $\pm 3,77^{\mathrm{b}}$ & 20,76 & 38,86 \\
G5 & 28,54 & $\pm 4,46^{\mathrm{b}}$ & 19,80 & 36,69 \\
G6 & 28,45 & $\pm 3,06^{\mathrm{b}}$ & 23,99 & 35,60 \\
G7 & 29,33 & $\pm 4,20^{\mathrm{b}}$ & 23,27 & 36,90 \\
G8 & 28,58 & $\pm 4,32^{\mathrm{b}}$ & 19,27 & 36,83 \\
G9 & 28,34 & $\pm 4,86^{\mathrm{b}}$ & 19,50 & 41,91 \\
G10 & 28,06 & $\pm 3,32^{\mathrm{b}}$ & 24,00 & 36,68 \\
\hline
\end{tabular}

TABELA 2 Média e desvio-padrão dos grupos avaliados por IMC.

Nota: Letras "a" e "b" representam diferença significativa $(p<0,05)$ após teste ANOVA two-way utilizando post-hoc Tukey test entre G1 e G2 e demais grupos; DP: desvio-padrão.

Quanto aos dados obtidos por meio da glicemia, estes apresentam limitações, visto que não foram realizados com todos os indivíduos em jejum, considerando hiperglicemia apenas indivíduos com valores acima de $200 \mathrm{mg} / \mathrm{dl}$, pela avaliação da glicemia capilar casual obtida por meio de glicosímetro portátil. No total 18 indivíduos foram avaliados com hiperglicemia, sendo refeito o teste para confirmar e orientados a procurar o serviço adequado para uma melhor avaliação, dos quais doze tinham sobrepeso, quatro obesos e dois eram eutrófico, sendo do total 13 do sexo masculino e cinco do sexo feminino. Todos com idade acima de 44 anos (tabela 3 ).

TABELA 3 Número de indivíduos por faixa de glicemia capilar.

\begin{tabular}{cccc}
\hline GRUPOS & $\mathbf{7 0 - 9 9}$ & $\mathbf{1 0 0 - 1 9 9}$ & $\geq 200$ \\
\hline G1 & 15 & 15 & 0 \\
G2 & 12 & 18 & 0 \\
G3 & 8 & 22 & 0 \\
G4 & 8 & 22 & 0 \\
G5 & 11 & 18 & 1 \\
G6 & 11 & 18 & 1 \\
G7 & 14 & 15 & 1 \\
G8 & 6 & 20 & 4 \\
G9 & 12 & 15 & 3 \\
G10 & 6 & 16 & 8 \\
TOTAL & $103(34,33 \%)$ & $179(59,66 \%)$ & $18(6,00 \%)$ \\
\hline
\end{tabular}

Os grupos com maior número de indivíduos com glicemia capilar acima de $200 \mathrm{mg} / \mathrm{dL}$ foram os grupos G8 (Masculino, entre 55 a 64 anos) e G10 (Masculino, 
igual ou acima de 65 anos) com quatro e oito indivíduos, respectivamente, representando dentro da amostra que indivíduos mais idosos foram os que tiveram maior hiperglicemia. Entretanto, é válido salientar que os grupos com maiores índices de massa corpórea foram G4 e G7. O percentual de indivíduos com diabetes segundo dados da VIGITEL em 2018 para pessoas acima de 18 anos foi de 4,8\% para mulheres e 7,6\% para homens no município de Manaus-AM (BRASIL, 2018).

Apesar da conexão entre diabetes e obesidade ser bem esclarecida, outros estudos envolvendo populações asiáticas demonstrou que os habitantes da região apresentam uma baixa frequência de obesidade e um elevado risco de diabetes em qualquer nível de índice de massa corporal em comparação àqueles que vivem nos Estados Unidos ou Europa, uma explicação apoiada é o achado de que os homens asiáticos, por exame de tomografia computadorizada abdominal, apresentam mais gordura visceral e menos gordura subcutânea do que os homens caucasianos (WU et al., 2017). Este trabalho demonstra que há outros fatores que podem estar relacionados com estes resultados que mostrou maior número de homens com hiperglicemia, em comparação às mulheres (tabela 4).

TABELA 4 Média e desvio-padrão dos grupos avaliados por glicemia capilar.

\begin{tabular}{ccccc}
\hline GRUPOS & MÉDIA & DP & MíNIMO & MÁXIMO \\
\hline G1 & 103,51 & $\pm 14,32^{\mathrm{a}}$ & 82 & 145 \\
G2 & 105,33 & $\pm 21,78^{\mathrm{a}}$ & 69 & 168 \\
G3 & 119,17 & $\pm 21,77^{\mathrm{a}}$ & 76 & 196 \\
G4 & 117,82 & $\pm 26,13^{\mathrm{a}}$ & 76 & 189 \\
G5 & 120,83 & $\pm 51,32^{\mathrm{a}}$ & 61 & 344 \\
G6 & 112,73 & $\pm 29,09^{\mathrm{a}}$ & 80 & 221 \\
G7 & 108,93 & $\pm 29,60^{\mathrm{a}}$ & 75 & 220 \\
G8 & 128,55 & $\pm 56,85^{\mathrm{a}}$ & 73 & 304 \\
G9 & 128,57 & $\pm 36,66^{\mathrm{a}}$ & 61 & 362 \\
G10 & 170,37 & $\pm 103,14^{\mathrm{b}}$ & 70 & 483
\end{tabular}

Nota: A letra "b" representa diferença significativa entre o Grupo 10 e os demais grupos $(p<0,05)$; DP: Desvio-padrão.

Estudos realizados na África subsaariana, levando em consideração diferenças entre gêneros, observaram assim como neste levantamento, que houve uma maior taxa entre os homens de níveis hiperglicêmicos, em proporção ao grupo de mulheres (HILAWE et al., 2013). Pesquisas demonstram que os homens possuem menor sensibilidade hepática à insulina e podem ter elevação maior nos níveis glicêmicos séricos, outra explicação seria a taxa de tabagismo entre os homens que também faz diminuir a sensibilidade à insulina. Entretanto, as mulheres possuem menor massa muscular corporal e altos níveis de progesterona e estrógeno, tais fatores também contribuem para a menor sensibilidade de insulina em todo o corpo. Dessa forma, os hábitos dos homens como alcoolismo, tabagismo, dieta hipercalórica e sedentarismo podem refletir nessas taxas maiores de hiperglicemia do que em mulheres (BURGIO et al., 2014). 
Torna-se cada vez mais evidente que a epidemia de obesidade não pode ser explicada simplesmente como resultado de uma ingestão excessiva de alimentos altamente calóricos e sedentarismo, mas um sistema complexo que envolve fatores genéticos, imunológicos e neuroendócrinos, resultante de um distúrbio sustentado na regulação do metabolismo energético que favorece 0 armazenamento de triglicerídeos e hipertrofia de adipócitos (ABRANCHES et al., 2015). Pesquisas demonstraram que o tecido adiposo não é meramente um local de armazenamento de lipídios, mas um sistema multifacetado que atua em diversas funções endócrinas e serve como regulador da homeostase energética do organismo (OKAMURA et al., 2018). Essas pesquisas deixam claro que nem todos os indivíduos com obesidade por muitos anos terão diabetes, pois há outros fatores envolvidos, visto que nessa pesquisa verificou-se que há muitos obesos com glicemia normal, assim como há pacientes com hiperglicemia e são eutróficos.

Essa correlação entre aumento do tecido adiposo e resistência à insulina nos indivíduos estudados nesta pesquisa (tabelas 2 e 4) pode ser fundamentada nos estudos realizados por Canfora et al. (2019), que demonstraram que durante o declínio metabólico associado a obesidade, os adipócitos param de responder adequadamente a quase todos os sinais extracelulares, principalmente à insulina, acarretando elevados níveis de glicose e lipídios circulantes, além disso há aumento de citocinas pró-inflamatórias, aumentando risco de doenças cardiovasculares e reumatológicas.

Certas adipocinas, tais como IL-1b (interleucina 1 beta), IL-6 (interleucina 6), IL-8 (interleucina 8), TNF-alfa (Fator de Necrose Tumoral-alfa) e quimiocinas contribuem juntas para desregulação metabólica observada na obesidade e síndrome metabólica. O TNF-alfa especificamente em níveis elevados contribui diretamente para a fosforilação do receptor de insulina, bloqueando a cascata de sinalização, consequentemente, favorecendo ao surgimento da resistência à insulina, sendo uma possível explicação para a maior taxa de hiperglicemia nos indivíduos desta amostra que tinham IMC elevado. Porém, é ainda um tema bastante discutido, visto que uma análise maior dos dados disponíveis demonstrou que as vias de sinalização da responsividade à insulina, incluindo a modulação do fluxo metabólico, regulação transcricional e outras vias, poderiam ser mais importantes do que a fosforilação na maioria dos indivíduos com obesidade e diabetes mellitus do tipo 2 (GHABEN; SCHERER, 2019).

Foi observada elevada taxa de hiperglicemia na amostra de indivíduos avaliada no estudo, principalmente entre os mais idosos, sugerindo uma relação entre envelhecimento e DM 2 (tabela 4). Tais constatações são corroboradas por uma pesquisa feita na China com 509 pacientes, todos com idade superior a 50 anos, na qual foi comparada a resistência a insulina entre os indivíduos, sendo que os resultados sugeriram que há um declínio da função das células beta pancreáticas em idosos, porém somente naqueles em que há um alto grau de resistência insulínica por mais tempo, os quais tinham perdido a capacidade de compensar os níveis de glicemia com o aumento da função das células beta (CAl et al., 2019). Uma outra explicação para os resultados seria que em análises do envelhecimento do pâncreas por meio de exames de imagem e laboratoriais foi visto que o volume do órgão sofre um declínio a partir de aproximadamente 60 anos, mas sem que haja doença pancreática endócrina, sendo as alterações pancreáticas na idade avançada relacionadas à aterosclerose de pequenos vasos (LOHR et al., 2018). 
Em um trabalho de coorte prospectivo com 4.252 indivíduos relacionando sarcopenia, DM 2 e obesidade, também verificou que os indivíduos mais idosos e sedentários, independentemente do índice de massa corpórea, tinham maior predisposição para o desenvolvimento de DM 2 e apontou a prática de atividade física diária como um importante fator protetor para evitar o surgimento de DM 2 (ATKINS et al., 2014), o que está de acordo com o este estudo o qual evidenciou que os indivíduos com hiperglicemia estavam sedentários.

O município de Manaus, segundo estimativa do IBGE para 2019, tem uma população de 2.182.763 pessoas (IBGE, 2019), com bons índices econômicos, porém há na cidade diversos problemas sociais que influenciam diretamente na qualidade de vida da maioria da população que vive em regiões da cidade sem acesso à áreas de lazer ou que sofrem com medo da violência, a qual inibe a prática de atividade física, pois os cidadãos têm medo até mesmo de realizar uma simples caminhada pelas ruas devido a violência (FREITAS; MORAES, 2016), soma-se a isso as dificuldades de acesso à alimentos mais nutritivos e saudáveis que são mais caros na cidade, como frutas e verduras.

De acordo com dados obtidos pelo inquérito telefônico da VIGITEL, o consumo de frutas e hortaliças é maior pelas mulheres, aproximadamente $28,8 \%$ consomem, enquanto apenas $24,8 \%$ dos homens de Manaus afirmam consumirem, evidenciando a preferência dos homens de Manaus por outros alimentos mais processados (BRASIL, 2018).

A logística para abastecer os mercados de Manaus reflete nos preços dos alimentos, sendo que frutas e hortaliças são mais caras do que em muitos estados, por este motivo a maior parte da população consome mais produtos ultraprocessados e/ou com alto teor de açúcares. Sendo importante capacitar os profissionais de saúde de diversas áreas, principalmente os que atendem em postos de saúde, para passar as informações básicas sobre os riscos de consumo de alimentos ultraprocessados e sugerir mudanças de acordo com as condições locais para uma alimentação mais saudável (FERREIRA et al., 2010).

A dificuldade em obter alimentos frescos acarreta maior busca por alimentos ultraprocessados que são vendidos em pequenas mercearias mais próximas de suas casas (FERREIRA et al., 2010), que têm elevado valor calórico, tais como embutidos e bebidas de alto valor energético contribuindo para o aumento da obesidade e, consequentemente, o surgimento de doenças secundárias na família.

Muitos bairros da cidade, devido ao crescimento desordenado da mesma, não têm a devida assistência de saúde, sendo prejudicados no sentido de não haver orientações quanto à alimentação e cuidados com a saúde, faltando o acompanhamento com nutricionista e educadores físicos. A cidade também tem deficiência em locais públicos para prática de exercícios físicos, como parques e malha cicloviária pequena (FREITAS; MORAES, 2016).

Por meio dos dados obtidos em 2016 pela VIGITEL foi observado que houve um aumento da taxa de prevalência de diabetes no Brasil de 5,5\% em 2006 para aproximadamente $8,0 \%$ em 2016, sendo o aumento mais pronunciado em homens $(0,28 \%$ por ano) do que em mulheres $(0,23 \%$ por ano). Indivíduos mais idosos (acima de 65 anos) e acima do peso (IMC $\geq 25$ ) tiveram taxas mais elevadas de diabetes (ISER et al., 2016), assim como na amostra obtida nesse estudo. 


\section{CONCLUSÃO}

No presente estudo verificamos que principalmente os mais idosos e do sexo masculino apresentaram o índice maior de obesidade e diabetes, também foi observado que há certa dificuldade para acesso a alimentação saudável, tanto pela disponibilidade quanto pelo custo. Portanto, isso evidencia a maior necessidade de incentivar a prática de exercícios físicos nessa idade, providenciar programas com educadores físicos em locais públicos com maior efetivo de segurança. É imprescindível, também, que os profissionais de unidades básicas de saúde orientem de forma clara e objetiva os pacientes sobre alimentação equilibrada levando em conta a realidade da comunidade na qual estão inseridos, incluindo os aspectos socioeconômicos. Além disso, o aumento da malha cicloviária da cidade se faz importante, pois também favorece a prática de atividade física ao ar livre, já que uma grande parcela da população não tem acesso a espaços privados, como academias.

\section{REFERÊNCIAS}

ABESO. Mapa da obesidade 2016. Seção científica. Disponível em < http://www.abeso.org.br/atitude-saudavel/mapa-obesidade/>. Acesso em 07 de junho de 2019.

ABRANCHES, M. V.; OLIVEIRA, F. C. E.; CONCEIÇÃO L.L.; PELUZIO M. D. Obesity and diabetes: the link between adipose tissue dysfunction and glucose homeostasis. Nutrition research reviews, v. 28, n. 2, p. 121-132, 2015. Disponível em: < http://dx.doi.org/10.1017/S0954422415000098>. doi: $10.1017 / \mathrm{S} 0954422415000098$.

ATKINS, J. L.; WHINCUP, P. H.; MORRIS, R.W.; LENNON, L. T.; PAPACOSTA, O. Sarcopenic obesity and risk of cardiovascular disease and mortality: A populationbased cohort study of older men. Journal of American Geriatrics Society, v. 62, n.2, p. 253-260, 2014. Disponível em: < https://dx.doi.org/10.1111/jgs.12652 > doi: $10.1111 /$ jgs. 12652 .

BRASIL. Ministério da Saúde. Vigitel Brasil, 2018: Vigilância de Fatores de Risco e Proteção para Doenças Crônicas por Inquérito Telefônico. Secretaria de Vigilância em Saúde. Brasília: Ministério da Saúde, 2018.

BURGIO, E.; LOPOMO, A.; MIGLIORE, L. Obesity and diabetes: from genetics to epigenetics. Molecular Biology Reports, v. 42, n. 4, p. 799-818, 2014. Disponível em: < http://dx.doi.org/10.1007/s11033-014-3751-z >. doi: 10.1007/s11033-014$3751-z$.

CAI, X.; XIA, L.; PAN, Y.; HE D.; ZHU, H.; et al. Differential role of insulin resistance and $\beta$-cell function in the development of prediabetes and diabetes in middle-aged and elderly Chinese population. Diabetology and Metabolic Syndrome, v. 11, n.1, p.1-8, 2019. Disponível em: <https://dx.doi.org/10.1186/s13098-019-0418-x> doi: 10.1186/s13098-019-0418-x. 
CANFORA, E. E; MEEX, R. C. R.; VENEMA, K.; BLAAK E. E. Gut microbial metabolites in obesity, NAFLD and T2DM. Nature Reviews Endocrinology, v. 15, n. 5, p. 261-273, 2019. Disponível em: <https://dx.doi.org/10.1038/s41574-0190156-z >. doi: 10.1038/s41574-019-0156-z.

FERREIRA, V. A.; SILVA, A. E.; RODRIGUES, C. A. A.; NUNES, N. L. A.; VIGATO, T. C.; et al. Desigualdade, pobreza e obesidade. Revista Ciência \& Saúde Coletiva, v. 15, supl. 1, p. 1423-1432, 2010. Disponível em: <http://dx.doi.org/10.1590/S1413-81232010000700053>. doi: 10.1590/S141381232010000700053.

FREITAS, I. C. M.; MORAES, S. A. O efeito da vulnerabilidade social sobre indicadores antropométricos de obesidade: resultados de estudo epidemiológico de base populacional. Revista brasileira de epidemiologia, São Paulo, v. 19, n. 2, p. 433-450, 2016. Disponível em: < http://dx.doi.org/10.1590/19805497201600020018. > . doi: 10.1590/1980-5497201600020018.

GHABEN, A. L.; SCHERER, P. E. Adipogenesis and metabolic health. Nature Reviews Molecular Cell Biology, v. 20, n. 4, p. 242-258, 2019. Disponível em: < http://dx.doi.org/ 10.1038/s41580-018-0093-z. > . doi: 10.1038/s41580-018-0093-z.

HILAWE, E. H.; YATSUYA, H.; KAWAGUCHI, L.; AOYAMA, A. Differences by sex in the prevalence of diabetes mellitus, impaired fast in glycaemia and impaired glucose tolerance in sub-Saharan Africa: a systematic review and meta-analysis. Bulletin of the World Health Organization, v. 91, n. 9, p. 671-682, 2013. Disponível em: <http://dx.doi.org/10.2471/BLT.12.113415>. doi: 10.2471/BLT.12.11341.

HRUBY, A.; HU, F. B. The epidemiology of obesity: a big picture. Pharmacoeconomics, v. 33, n. 7, p. 673-689, 2015. Disponível em: < http://dx.doi.org/ 10.1007/s40273-014-0243-x > . doi: 10.1007/s40273-014-0243-x.

HSIA, D.S.; HEYMSFIELD, S.B. Reducing diabetes risk at na early age. Nature medicine, v. 24, n. 6, p. 708-710, 2018. Disponível em: < http://dx.doi.org /10.1038/s41591-018-0067-x>. doi: 10.1038/s41591-018-0067-x.

IBGE. Instituto Brasileiro de Geografia e Estatistica- Portal IBGE cidades 2019. Disponível em < https://cidades.ibge.gov.br/brasil/am/manaus/panorama/>. Acesso em 16 de outubro de 2019.

ISER, B. P. M.; VIGO, A.; DUNCAN, B.B.; SCHIMIDT, M. I. Trends in the prevalence of self-reported diabetes in Brazilian capital cities and the Federal District, 20062014. Diabetology and Metabolic Syndrome, v. 8, n. 70, p. 76-81, 2016. Disponível em: < http://dx.doi.org/10.1186/s13098-016-0185-x>. doi: 10.1186/s13098-0160185-x.

LOHR, J. M.; PANIC, L.; VUJASINOVIC, M.; VERBEKE, C. S. The ageing pancreas: a systematic review of the evidence and analysis of the consequences. Journal of 
Internal Medicine, v.283, n. 5, p 446-460, 2018. Disponível em < http://dx.doi.org/ 10.1111/joim.12745>. doi: 10.1111/joim.12745.

OKAMURA, T.; HASHIMOTO, Y.; HAMAGUCHI, M.; OBORA, A.; KOJIMA, T.; et al. Ectopic fat obesity presents the greate strisk for incident type 2 diabetes: a population-based longitudinal study. International Journal of Obesity, v. 43, n.1, p. 139-148, 2018. Disponível em: < http://dx.doi.org/10.1038/s41366-018-0076-3 >. doi: 10.1038/s41366-018-0076-3.

SCHWARTZ, M. W.; SEELEY, R. J.; ZELTSER, L. M.; DREWNOWSKI, A.; RAVUSSIN, E.; et al. Obesity pathogenesis: an Endocrine Society scientific statement. Endocrine reviews, v. 38, n. 4, p. 267-296, 2017. Disponível em: < http://dx.doi.org/10.1210/er.2017-00111 >. doi: 10.1210/er.2017-00111.

WHO - Organização Mundial de Saúde. Physical status: the use and interpretation of anthropometry. Report of a WHO Expert Committee. WHO Technical Report Series 854, 1995.

WU, J.; GONG, L.; QIFU, L.; HU, J.; ZHANG, S.; et al. A Novel Visceral Adiposity Index for Prediction of Type 2 Diabetes and Pre-diabetes in Chinese adults: A 5-year prospective study. Scientific Reports, v. 7, n. 13784, p. 1-9, 2017. Disponível em: < http://dx.doi.org/10.1038/s41598-017-14251-w >. doi: 10.1038/s41598-017-14251-w. 